\title{
ANÁLISE DO GERENCIAMENTO DOS RESÍDUOS SÓLIDOS DE SERVIÇOS DE SAÚDE: O CASO DE UM HOSPITAL DE MÉDIO PORTE DO INTERIOR DO ESTADO DE SÃO PAULO
}

\section{Edenis César de Oliveira*}

Resumo. É amplamente reconhecida a importância do manejo adequado dos resíduos sólidos como condição necessária ao bem estar de toda população, incluído o cuidado com o meio ambiente, além do aspecto da saúde pública em geral. Nesse contexto, os resíduos sólidos de serviços de saúde ganham atenção especial, notadamente por se tratar de resíduos que podem oferecer riscos diretos à saúde da comunidade se não forem seguidas as regras corretas de manejo. Nessa perspectiva, o presente trabalho apresenta, a partir de uma revisão bibliográfica e utilização da técnica do Estudo de Caso, um diagnóstico do processo de gerenciamento dos RSS num hospital de médio porte do interior do Estado de São Paulo. É conclusiva deste trabalho a importância estratégica de um Plano de Gerenciamento de Resíduos Sólidos de Serviços de Saúde - PGRSS para uma instituição hospitalar, sobretudo como instrumento norteador das ações básicas para o correto manejo dos seus resíduos com vistas à qualidade dos serviços de atenção direta ao cliente/paciente.

\section{INTRODUÇÃO}

É sabido que a geração de resíduos provenientes das atividades humanas faz parte da própria história da humanidade. É a partir da segunda metade do século XX, com os novos padrões de consumo da sociedade industrializada, que se apresenta um crescimento exponencial, num ritmo superior à capacidade de absorção pela natureza. Aliado a isso, o avanço tecnológico das últimas décadas, se, por um lado, possibilitou

\footnotetext{
*Administrador de Empresas; Especialista em Gestão Empresarial e Mestre em Planejamento e Gestão Ambiental pela FCT/UNESP - Presidente Prudente/SP. Coordenador do Curso de Administração da Fundação Gammon de Ensino (FUNGE).e-mail: edenis@netonne.com.br
} 
conquistas e avanços surpreendentes no campo da ciência, por outro, contribuiu para o aumento da diversidade de produtos com componentes e materiais de difícil degradação e maior toxidade (MS/ANVISA, 2006).

A preocupação com as questões ambientais está no centro das decisões estratégicas das organizações empresariais, acentuadamente nesta primeira década deste novo século. Dessa forma, todo resíduo gerado por essas empresas devem, necessariamente, ter um destino adequado, seja com o reaproveitamento, com a reciclagem, ou quando não for possível nestes casos, a garantia da correta destinação.

Consoante Pereira e Tocchetto (2004) ao gerenciar de forma correta a empresa estará demonstrando à sociedade seu nível de compromisso com o meio ambiente através da inserção de mudança organizacional estratégica evidenciada na internalização ambiental e externalização de práticas que integram o meio ambiente e a produção de serviços, além de outros objetivos como a melhoria de sua própria imagem, maior interação com o empreendimento, minimização dos riscos de infrações e multas, entre outros.

De acordo com o disposto em IBAM (2001, p. 8):

Pode-se considerar o gerenciamento integrado do lixo quando existir uma estreita interligação entre as ações normativas, operacionais, financeiras e de planejamento das atividades do sistema de limpeza urbana, bem como tais articulações se manifestarem também no âmbito das ações de limpeza urbana com as demais políticas públicas setoriais. Nesse cenário, a participação da população ocupará papel de significativo destaque, tendo reconhecida sua função de agente transformador no contexto da limpeza urbana.

$\mathrm{Na}$ classificação de origem dos resíduos sólidos em geral, os resíduos sólidos de serviços de saúde (RSS) enquadram-se na categoria de fontes especiais e, portanto, devem receber o tratamento adequado conforme dispõe as normativas próprias.

Takayanagui (2005) observa que até o final da década de 1980, os RSS eram denominados lixo hospitalar. Em 1987 a Associação Brasileira de Normas Técnicas alterou a terminologia para resíduo de serviço de saúde, embora deva-se considerar que 
esse tipo de resíduo não é exclusivamente gerado em hospitais mas, também, provém de ambulatórios, consultórios médicos e odontológicos, clínicas veterinárias, farmácias, laboratórios de análises clínicas e patológicas, bancos de sangue ou leite, além de estações rodo-ferroviárias, portos e aeroportos e locais de grande afluxo de pessoas (ABNT, 1993).

No entender de Brito (2000), as instituições de saúde não podem mais "cuidar" do lixo apenas do ponto de vista do controle de infecção, mas precisam também considerar as questões ambientais, o que envolve questionamentos dos hábitos, costumes, análise de fatores econômicos e culturais envolvidos.

Dentro de uma organização hospitalar, a questão ambiental assume variadas dimensões, sendo todas elas muito importantes, complexas e dignas de um tratamento sério e sistêmico em seu conjunto. No entanto, é inegável a emergência e a criticidade da gestão dos resíduos sólidos de uma unidade hospitalar, sobretudo pelo próprio crescimento na produção desses resíduos, provocado, principalmente pelo incremento da complexidade dos procedimentos, a universalização do sistema, bem como, o uso crescente de materiais descartáveis (SANCHES, 1995).

Em se tratando de uma organização de saúde, no caso uma unidade hospitalar, a atenção aos resíduos por ela gerados deve receber um tratamento especial, uma vez que se trata de resíduos com certas especificidades, que podem causar riscos de contaminação, com agravos diretos à saúde do trabalhador e da sociedade, além de outros prejuízos ao meio ambiente.

Para Catão et al (2007) principalmente pela falta ou pouca clareza das informações, os RSS ou "lixo hospitalar" como é mais comumente denominado, constituem-se num problema bastante sério para os administradores hospitalares. Os mesmos autores acrescentam que "os danos provocados ao meio ambiente pelo lixo hospitalar não são poucos, pois substâncias radioativas e quimioterápicas, anineoplásticos são jogados indiscriminadamente nos aterros onde se encontra o lixo comum, causando as mais deletérias conseqüências. [...] não só são afetados aqueles que vivem da coleta do lixo ou moram próximo aos aterros, mas também os funcionários 
que, diariamente e desprotegidos, estão em contato direto com a invisível carga radioativa" (p. 23).

Tal assertiva pode ser confirmada por Naime et al (2008, p. 2) ao considerar que "as questões relacionadas aos aspectos ambientais em uma instituição hospitalar são de relevância considerável no contexto da manutenção da qualidade de vida de uma sociedade".

No Brasil, são geradas cerca de 120 mil toneladas de lixo urbano por dia. Um a três por cento desse total é produzido nos estabelecimentos de saúde e, destes, $10 \%$ a $25 \%$ representam risco ao meio ambiente e a saúde da população (ANVISA, 2004).

Importante ressaltar que nem todo resíduo gerado nas dependências de uma unidade hospitalar são considerados como perigosos ou infectantes. A inobservância desta questão pode levar a organização a desprender recursos além do necessário no tratamento dos seus resíduos.

Para Silva (1993), dentre os resíduos gerados num hospital, constituem-se em motivo de preocupação, por representarem risco potencial à saúde pública, os resíduos radioativos, os químicos, os farmacêuticos, os perfurocortantes e aqueles contaminados por microrganismos patogênicos.

Consoante Ferreira (1995), os RSS podem ser considerados de natureza heterogênea e, mais especificamente no caso de uma unidade prestadora de serviço hospitalar, podem ser discriminados em: lixo comum (papel, restos de jardim, restos de comida de refeitórios e cozinhas), resíduos infectantes ou de risco biológico (sangue, gaze, curativo e agulha) e resíduos especiais (químicos, farmacêuticos e radioativos).

A Agência Nacional de Vigilância Sanitária através da RDC no 306 (ANVISA, 2004), se encarrega do regulamento técnico para o gerenciamento dos resíduos de serviços de saúde.

\section{0 gerenciamento dos resíduos sólidos de serviços de saúde}


Considera-se o gerenciamento dos resíduos de serviços de saúde um assunto polêmico embora amplamente discutido por tratar-se de lixo hospitalar. Produzidos em todos os estágios das atividades humanas, os resíduos, em termos tanto de composição como de volume, variam em função das práticas de consumo e dos métodos de produção. As principais preocupações estão voltadas para os efeitos que podem ter sobre a saúde humana em particular e sobre o meio ambiente em geral (CAMARGO et al, 2009).

O gerenciamento dos resíduos de serviços de saúde (RSS) constitui-se em um conjunto de procedimentos de gestão, planejados e implementados a partir de bases científicas e técnicas, normativa e legais, com o objetivo de minimizar a produção de resíduos e proporcionar aos resíduos gerados, um encaminhamento seguro, de forma eficiente, visando à proteção dos trabalhadores, a preservação da saúde pública, dos recursos naturais e do meio ambiente (ANVISA, 2004).

Nesse contexto, destaca-se a importância do Plano de Gerenciamento de Resíduos Sólidos de Saúde (PGRSS) dentro de uma unidade hospitalar, como importante ferramenta de gestão para garantir a minimização dos custos e, concomitantemente, a garantia do atendimento às normas legais, além do mais importante, a garantia da saúde do trabalhador, do bem-estar da comunidade e do meio ambiente em geral.

As etapas do gerenciamento dos RSS estão contempladas dentro do processo de MANEJO, entendido como a ação de gerenciar os resíduos em seus aspectos intra e extra estabelecimento, desde a geração até a disposição final, incluindo as seguintes etapas: segregação, acondicionamento, identificação, transporte interno, armazenamento temporário, tratamento, armazenamento externo, coleta e transporte externos e disposição final.

No entendimento de Teixeira (2006), a gestão dos RSS ainda apresenta certa complexidade em função da limitada experiência das administrações municipais em atuar com eficiência neste campo dos resíduos sólidos, agravado pela grande quantidade de normas e regulamentações sobre o tema. 
A falta de experiência por parte das administrações públicas municipais, agravada pela falta de profissionais técnicos qualificados, somada às informações complexas, difusas e às vezes conflitantes das normas e legislações, possibilitou que a questão do gerenciamento dos resíduos sólidos em geral e dos resíduos sólidos de saúde (RSS) em particular fossem deixados para um segundo plano.

Entretanto, com a forte valorização das questões ambientais, aqui compreendidas o cuidado com o meio ambiente, agregado à responsabilidade sócio-ambiental dos tomadores de decisão, em especial, a legislação pertinente sofreu atualização, permitindo maior clareza na elaboração dos planos de gerenciamento e, portanto nas ações propriamente ditas.

Por outro lado, é pertinente a questão proposta por Marangoni (2006, p. 38), ao afirmar que "a gestão de RSS deve contemplar todos os aspectos envolvidos, desde a fonte geradora até a disposição segura, bem como a máxima minimização e educação, buscando inclusive incorporar mudanças nos indivíduos quanto à produção e consumo".

Reconhecidamente este é o caminho que se deve trilhar quando se busca implementar mudanças profundas e duradouras. A adequação e atualização das normativas, o esforço para o enquadramento na legalidade por parte das fontes geradoras e, paralelamente, a mudança de hábitos e educação continuada de todos os atores envolvidos.

Importantes princípios como reduzir, segregar e reciclar devem orientar o gerenciamento dos resíduos, sendo incorporados ao Plano de Gerenciamento de Resíduos Sólidos de Serviços de Saúde (PGRSS) de todos os estabelecimentos geradores (GARCIA e ZANETTI-RAMOS, 2004).

Ferreira (1995) salienta que a classificação dos resíduos é uma atividade complexa e, em muitos casos, ainda indefinida mesmo nos países desenvolvidos. Quanto mais perigoso for considerado o resíduo, maiores os cuidados necessários e, como conseqüência, maiores os custos envolvidos.

Por conseguinte, Garcia e Zanetti-Ramos (2004) alertam para o fato de que a etapa da segregação constitui-se num ponto crucial sobre a discussão da periculosidade dos 
resíduos de serviços de saúde. Apenas uma parte apresenta-se como potencialmente infectante, exigindo procedimentos especiais para acondicionamento, coleta, transporte e disposição final, elevando assim, os custos do tratamento desses resíduos.

Nessa perspectiva, Lippel (2003) acrescenta que a segregação no momento e local da geração dos resíduos, permite reduzir o volume daqueles considerados perigosos, além da incidência de acidentes ocupacionais dentre outros benefícios à saúde pública e ao meio ambiente.

Os principais objetivos da segregação são:

- Minimizar a contaminação de resíduos considerados comuns;

- Permitir a adoção de procedimentos específicos para o manejo de cada grupo de resíduos;

- Possibilitar o tratamento específico para cada categoria de resíduo;

- Reduzir os riscos para a saúde;

- Diminuir os custos no manejo dos resíduos;

- Reciclar ou aproveitar parte dos resíduos comuns.

Para o correto desempenho dessa importante etapa (segregação dos resíduos), os autores em questão, reforçam a necessidade de investimento na capacitação dos colaboradores envolvidos, o que resultará em redução das despesas com tratamento ao mínimo necessário.

O cumprimento a contento desta etapa, possibilitará condições de encaminhamento de parte dos resíduos para a reciclagem (por exemplo: embalagens, material de escritório, papel, papelão, plásticos de uso comum, entre outros), inserindo tais materiais, novamente, no ciclo produtivo.

No entender de Garcia e Zanetti-Ramos (2004, p. 6) "o processo de reciclagem traz benefícios para a comunidade, pois gera empregos e renda, além de contribuir para a redução da poluição ambiental, pois menos resíduos são depositados em aterros, e ainda implica em menor gasto de recursos naturais". 


\section{Diagnóstico de gerenciamento dos resíduos sólidos de serviços de saúde do Hospital de Médio Porte.}

O Hospital de Médio Porte (HMP), objeto de estudo deste trabalho, é uma organização hospitalar privada, de caráter beneficente e filantrópico, fundada há mais de 60 anos, sendo o único hospital no município. Conta com 102 leitos (Tabela 1) e 154 funcionários, além de um Corpo Clínico com aproximadamente 40 profissionais médicos.

Tabela 1 - № de leitos distribuídos por clínicas do HMP

\begin{tabular}{lc}
\multicolumn{1}{c}{ Setor } & No de Leitos \\
\hline Clínica Médica & 24 \\
Pediatria & 15 \\
UTI - Unidade de Terapia Intensiva & 05 \\
Pronto Atendimento (observação) & 10 \\
Clínica Cirúrgica & 26 \\
Maternidade & 22 \\
\hline Total & $\mathbf{1 0 2}$ \\
\hline
\end{tabular}

Fonte: elaborado pelo autor com base no CNES - Cadastro Nacional de Estabelecimento de Saúde.

A instituição hospitalar possui uma área construída de $5.679,02 \mathrm{~m}^{2}$, situada num terreno com área total de $12.104,50 \mathrm{~m}^{2}$, tendo $6.424,48 \mathrm{~m}^{2}$ de área livre.

O Hospital é conveniado ao Sistema Único de Saúde - SUS, de onde se origina aproximadamente $85 \%$ de sua demanda de trabalho. O restante destina-se principalmente aos convênios e uma quantidade pouco expressiva ao atendimento de particulares.

Muito embora resíduos sólidos de serviços de saúde (RSS) sejam definidos como aqueles provenientes de qualquer unidade que execute atividades de natureza médicoassistencial humana ou animal, aqueles provenientes de centros de pesquisa, desenvolvimento ou experimentação na área e farmacologia e saúde; medicamentos e imunoterápicos vencidos ou deteriorados; aqueles provenientes de necrotérios, funerárias, serviços de medicina legal e aqueles provenientes de barreiras sanitárias (AMANTHEA ET AL, 2005; ANVISA, 2004), para o fim a que se propõe este trabalho nos 
limitaremos aos resíduos sólidos de serviços de saúde oriundos de uma unidade hospitalar conforme descrito anteriormente.

No Hospital de Médio Porte (HMP) são oferecidas as especialidades de ginecologia/obstetrícia, pediatria, clínica médica, clínica cirúrgica, anestesiologia, traumaortopedia, cardiologia, serviço de nutrição e dietética - SND, diagnóstico por imagem (raio-x, ultrassonografia e tomografia computadorizada), além do serviço de urgência/emergência 24h, incluindo atendimento ambulatorial, Unidade de Terapia Intensiva - UTI com 10 leitos (5 leitos em funcionamento).

Os serviços de higiene, limpeza, coleta e destino interno dos resíduos do hospital são realizados pelos próprios funcionários lotados no setor de higiene e limpeza (13 funcionários no total). Na categoria de serviço de diagnóstico por imagem, a tomografia computadorizada é resultado de uma parceria entre o hospital e uma empresa privada.

Segundo proposto pela OPAS (1997), o gerenciamento correto dos resíduos sólidos significa não só controlar e diminuir os riscos, mas também alcançar a minimização dos resíduos desde o ponto de origem, que elevaria também a qualidade e a eficiência dos serviços que proporciona o estabelecimento de saúde.

Este mesmo guia, publicado pela Organização Pan-Americana de Saúde propõe, dentro dos aspectos organizacionais, entre outras, duas ações importantes que são: a) subdividir o estabelecimento de saúde de acordo com os serviços especializados; b) estabelecer uma classificação dos resíduos sólidos gerados (OPAS, 1997).

Os tipos de resíduos encontrados no hospital separados por setor podem ser vistos na tabela a seguir:

Tabela 2. - Tipos de resíduos originados por setor no HMP.

\begin{tabular}{l|l}
\multicolumn{1}{c}{$\begin{array}{c}\text { LOCAL } \\
\text { (Unidade ou Setor) }\end{array}$} & \multicolumn{1}{c}{ TIPOS DE RESíDUOS } \\
\hline ENFERMARIA & $\begin{array}{l}\text { Embalagens vazias de soro, seringas, scalps, agulhas, algodão, gazes, } \\
\text { esparadrapo, fita adesiva, papel, plástico, vidros, sondas, bolsas coletoras. }\end{array}$ \\
\hline $\begin{array}{l}\text { AMBULATÓRIO (Pronto } \\
\text { Atendimento) }\end{array}$ & $\begin{array}{l}\text { Papel, plástico, luvas, gazes, esparadrapo, algodão, espátulas, sondas, } \\
\text { scalps, bolsas coletoras, peças de gesso e ataduras (desde que não } \\
\text { estejam sujas de sangue ou secreções). }\end{array}$ \\
\hline LABORATÓRIO & $\begin{array}{l}\text { Lâminas, agulhas, seringas, recipientes, scalps, esparadrapo, algodão, } \\
\text { luvas, papel, plástico. }\end{array}$ \\
\hline
\end{tabular}




\begin{tabular}{l|l}
\hline LAVANDERIA & Luvas, papel, restos de tecido, efluentes líquidos. \\
\hline SND (COZINHA E COPA) & $\begin{array}{l}\text { Pratos de alumínio descartáveis, matéria orgânica, plásticos de } \\
\text { embalagens, papel. }\end{array}$ \\
\hline ADMINISTRATIVO & Papel, papelão e plástico. \\
\hline $\begin{array}{l}\text { UTI - Unidade de Terapia } \\
\text { Intensiva }\end{array}$ & $\begin{array}{l}\text { Frascos de soro, seringas, algodão, gazes, esparadrapo, fita adesiva, } \\
\text { papel, plástico, vidros, sondas, scalps, cateter de diálise, dispositivos } \\
\text { intravenosos (agulhas, escalpes, gelco, intracts), bolsas coletoras. }\end{array}$ \\
\hline CC - Centro Cirúrgico & $\begin{array}{l}\text { Embalagens vazias de soro, dispositivos intravenosos (agulhas, intracats, } \\
\text { scalps, gelco), gazes, esparadrapo, algodão, fita adesiva, papel, plástico, } \\
\text { vidros, sondas, bolsas coletoras, placenta e outras peças anatômicas } \\
\text { humanas. }\end{array}$ \\
\hline PÁTIO & Resíduos de varrição (folhagens), restos de poda, plásticos, papel. \\
\hline
\end{tabular}

Fonte: Elaborado pelo autor a partir dos dados da pesquisa de campo.

* Os efluentes líquidos provenientes das máquinas de lavar são destinado diretamente ao esgotamento sanitário, não recebendo nenhum tratamento especial.

Os serviços, por razões de risco sanitário, devem estar delimitados fisicamente no interior dos estabelecimentos de saúde. Esta delimitação física determinará, também, as fontes geradoras de resíduos.

Armazenamento temporário consiste na guarda temporária dos recipientes contendo os resíduos já condicionados, em local próximos ao ponto de geração, visando agilizar a coleta dentro do estabelecimento e otimizar o deslocamento entre os pontos geradores e o ponto destinado à apresentação para coleta externa (ANVISA, 2004).

Segundo o preconizado na RDC no 306/04 não poderá ser feito armazenamento temporário com disposição direta dos sacos sobre o piso, sendo obrigatória a conservação dos sacos em recipientes de acondicionamento. Neste quesito a Unidade atende as exigências, uma vez que os recipientes não estão dispostos diretamente sobre o piso.

A unidade hospitalar possui cinco (05) locais de armazenamento temporário interno os quais recebem o nome de expurgo (despejo), sendo 01 despejo para atender a Ala Pediátrica e Clínica Médica; 01 despejo para o Pronto Atendimento (PA); 01 despejo na Maternidade; 01 despejo na Ala 100 (destinada a convênios e particulares); e 01 despejo na Unidade de Terapia Intensiva (UTI). As salas utilizadas para o armazenamento temporário não se destinam exclusivamente ao armazenamento de resíduos, portanto, 
não há necessidade de identificação como "SALA DE RESÍDUOS", segundo a RDC n 306/04, item 1.5.2.

De acordo com Costa e Fonseca (2009), “armazenar os resíduos temporariamente em um local torna possível uma sistemática de transporte para o local de armazenamento externo" (p. 29). Ainda estes autores entendem que desta forma o contato dos resíduos com pacientes, acompanhantes e outras pessoas é evitado, impedindo contaminação.

Segundo informações obtidas com a Coordenadoria Técnica de Enfermagem, o Centro Cirúrgico não possui posto intermediário (despejo), sendo os resíduos levados diretamente ao armazenamento externo. As peças anatômicas oriundas do Centro Cirúrgico são encaminhadas diretamente para o laboratório de anatomocitopatologia (serviço terceirizado), não permanecendo, portanto, na instituição.

Além dos resíduos oriundos do Centro Cirúrgico, os da cozinha, laboratório, administrativo, lavanderia e pátio também seguem diretamente para o armazenamento externo.

A tabela seguinte sintetiza os tipos de resíduos originados no HMP bem como suas principais fontes de origem:

Tabela 3. - Origem dos resíduos separados por tipo de RSS

\begin{tabular}{l|l}
\hline \multicolumn{1}{c}{ TIPO DE RSS } & \multicolumn{1}{c}{ ORIGEM } \\
\hline INFECTANTE & $\begin{array}{l}\text { Enfermarias, Ambulatório, UTI, Laboratório, } \\
\text { Copa/Cozinha (no caso de sobras de alimentos de } \\
\text { quarto isolamento) }\end{array}$ \\
\hline QUÍMICOS & \begin{tabular}{l} 
Raio-X (resíduos químicos: fixador e revelador) \\
\hline RADIOATIVOS
\end{tabular} \\
\hline PERFUROCORTANTES & $\begin{array}{l}\text { Inexistente } \\
\text { Cirúrgico }\end{array}$ \\
\hline COMUNS & Administrativo, Ambulatório, Copa/Cozinha \\
\hline
\end{tabular}

Fonte: Elaborado pelo autor.

* Os resíduos provenientes do laboratório de radiologia são vendidos para empresa especializada que faz a coleta do material.

O hospital considera lixo comum os resíduos oriundos dos banheiros dos quartos dos pacientes (fraldas descartáveis, absorventes, papel higiênico etc.), não fazendo, 
também, nenhuma diferenciação dos resíduos que se originam dos quartos de isolamento, incluindo os de banheiro e de cozinha (orgânicos, pratos e copos descartáveis, entre outros).

O HMP condiciona os RSS de toda unidade em 3 embalagens distintas, a saber: resíduo infectante (sacos plástico de cor branca); resíduos perfurocortantes (caixas de papelão Descarpack); resíduos comuns (sacos plástico de cor preta). Segundo informação obtida em entrevista direta com Coordenadoria Técnica de Enfermagem, a instituição de saúde não promove a separação dos resíduos para reciclagem.

$\mathrm{Na}$ tabela seguinte estão sintetizadas as informações para melhor visualização.

Tabela 4. - Tipos de embalagens adotadas pelo HMP.

\begin{tabular}{l|l}
\hline \multicolumn{1}{c|}{ CATEGORIAS DE RSS } & \multicolumn{1}{c}{ TIPO DE EMBALAGEM } \\
\hline INFECTANTE & Saco plástico na cor branca \\
\hline PERFUROCORTANTE & Caixas de Papelão mod. Descarpack \\
\hline COMUM & Saco plástico na cor preta \\
\hline QUÍMICO & Galão de Plástico \\
\hline
\end{tabular}

Fonte: Elaborado pelo autor.

Na segunda visita à instituição com o objetivo precípuo de levantar informações e obter dados para subsidiar a continuidade da pesquisa, entrevistamos a profissional de enfermagem que acumula também o cargo de gerência do setor de higiene e limpeza do hospital.

Acompanhado desta profissional percorreu-se todos os setores da instituição, observando o fluxo interno dos resíduos. Em cada um dos postos intermediários de armazenamento interno, chamados na instituição de expurgos ou despejos, observou-se a disposição de 01 recipiente para resíduo comum (saco plástico preto), 01 recipiente para lixo contaminado (saco plástico branco) e 01 recipiente para perfurocortantes (caixas de papelão "descarpack").

Nos postos de enfermagem, há somente 01 recipiente para lixo comum (saco plástico preto) e 01 recipiente para perfurocortantes (descarpack). 
A partir destas observações, foi possível indagar a profissional que nos acompanhava sobre: a) O motivo da inexistência de recipiente para resíduo contaminado (saco plástico branco) nos postos de enfermagem; b) O motivo da existência de recipientes para resíduos perfurocortantes (caixas descarpack) nos despejos.

Para o primeiro questionamento, a resposta foi pelo custo do produto (embalagem saco branco), ou seja, por ser mais caro, mantêm-se somente nos despejos. Para a segunda pergunta, observou-se uma estratégia da instituição, pois segundo a profissional, "optou-se por deixar no despejo, também, uma embalagem para perfurocortantes, uma vez que, o profissional de enfermagem após sair do quarto com a bandeja de material já utilizado no paciente, pode ir direto para o despejo descartar o lixo contaminado e o perfurocortante, sem a necessidade de retornar ao posto de enfermagem para utilizar 0 recipiente descarpack".

Na Unidade de Terapia Intensiva (UTI) é disponibilizado, ao lado de cada leito, recipiente para resíduo contaminado (saco plástico branco), sendo recolhido diariamente e encaminhado ao despejo da própria Unidade e, posteriormente ao armazenamento externo.

Ainda, como resultado da observação in loco, constatou-se a ausência de recipiente para resíduo comum (saco plástico preto) nos despejos da Clínica Pediátrica / Clínica Médica e ala 100. Além disso, após verificação do conteúdo do recipiente para resíduo contaminado (saco plástico branco) do despejo do Ambulatório (Pronto Atendimento), constatou-se resíduo comum (papel, papelão e plástico) misturado com resíduos contaminados.

Observou-se também, que não há identificação na porta da sala de armazenamento externo dos resíduos, indicando que aquele local é para uso exclusivo de depósito de resíduo hospitalar infectante. Os recipientes são depositados diretamente no piso da sala de lixo infectante, tanto os sacos brancos como as caixas contendo os perfurocortantes. Além disso, a instituição não utiliza carros próprios para o transporte dos resíduos, sendo improvisado no próprio carrinho utilizado pela equipe de limpeza. 
Em conformidade com o que determina o MINISTÉRIO DA SAÚDE (2002), através de uma metodologia de caracterização que inclui avaliação qualitativa (composição) e quantitativa (quantidade atual e projetada), procedeu-se a pesagem dos resíduos por um período de sete (07) dias consecutivos, separando-os por unidades geradoras. Utilizou-se como instrumento de aferição uma Balança da Marca Filizola, Mod. 31, com capacidade máxima de $150 \mathrm{Kg}$ e precisão de $0,1 \mathrm{~kg}$.

Durante o processo de pesagem, através de observação do conteúdo dos recipientes, observou-se grande quantidade de resíduos comuns (principalmente papel e papelão) descartado no recipiente de lixo infectante, corroborando a ocorrência já constatada no armazenamento temporário do ambulatório, conforme anteriormente mencionado.

Os valores médios por setor de origem (fonte geradora) foram sintetizados na tabela seguinte para melhor visualização, bem como na figura subseqüente o percentual correspondente de cada tipo de resíduo.

Tabela 5. - Quantidade média diária de resíduos gerada por setor no HMP no período apurado

\begin{tabular}{|c|c|c|c|}
\hline \multirow{3}{*}{ Setor de origem } & \multicolumn{3}{|c|}{ Tipos de resíduos } \\
\hline & \multicolumn{3}{|c|}{ Valores médios diários (kg) } \\
\hline & Comum (Grupo D) & Infectante (Grupo A) & $\begin{array}{c}\text { Perfurocortante (Grupo } \\
\text { E) }\end{array}$ \\
\hline Enfermarias & 20,18 & 15,07 & 2,58 \\
\hline SND (Copa/Cozinha) & 43,85 & - & - \\
\hline Ambulatório & 7,87 & 13,3 & 2,85 \\
\hline Centro Cirúrgico & 1,36 & 5,85 & 0,80 \\
\hline UTI & 1,25 & 5,86 & 0,80 \\
\hline Laboratório & 0,39 & - & - \\
\hline Administrativo & 8,88 & - & - \\
\hline Lavanderia & 2,38 & - & - \\
\hline Pátio & 8,64 & - & - \\
\hline Total (média) & 94,80 & 40,08 & 7,03 \\
\hline
\end{tabular}

Fonte: Elaborador pelo autor.

Não houve quantidade suficiente de material perfurocortante no Laboratório durante o período, uma vez que, o recipiente é lacrado e disponibilizado no armazenamento externo somente quando está completamente cheio. Quanto aos resíduos infectantes gerados no Laboratório, estes são descartados nos postos de 
armazenamento intermediário das Enfermarias da unidade hospitalar, não tendo, portanto, recipiente próprio para descartar este tipo de resíduo. O Serviço de Nutrição e Dietética (SND) não promove a separação dos resíduos oriundos de quartos de pacientes que estejam em sistema de isolamento, portanto, não houve possibilidade de aferir estes valores, sendo incorporados na categoria de resíduos comuns.

\section{Considerações Finais}

Apesar da inexistência de um Plano de Gerenciamento de Resíduos Sólidos de Serviços de Saúde (PGRSS), a instituição hospitalar tem se mostrado, de certa forma, atenta a questões consideradas cruciais no manejo de seus resíduos. Todavia, alguns pontos considerados estratégicos devem ser observados para melhor adequação à legislação e, ainda, sua consolidação no compromisso com o meio ambiente.

A proposta de elaboração de um plano formal de gerenciamento envolvendo todos os colaboradores seria um ponto de partida fundamental para o caminho que a instituição pretende trilhar. O processo inicial de sensibilização de toda comunidade interna para a importância do manejo correto dos resíduos, seria de grande valia, acompanhado de um processo contínuo de capacitação (educação permanente?).

Entre as principais sugestões destacam-se as seguintes:

- Envolvimento de toda comunidade interna, além de representantes externos (clientes/fornecedores/parceiros) no processo de elaboração do Plano;

- Criação de Comissão específica para o desenvolvimento, implementação e monitoramento do Plano;

- Realização das adequações necessárias, levando-se em consideração a legislação pertinente, atualizada e em vigência;

- Aprimoramento do processo de segregação como importante etapa no processo em geral; 
- Separação dos resíduos para reciclagem com vistas ao fortalecimento da consciência ambiental dos diretamente envolvidos, além de servir de exemplo para a comunidade externa;

- Implementação de cursos regulares como parte do processo de treinamento/capacitação de todos os colaboradores.

- Adequação da sala de armazenamento externo, tanto para os resíduos infectantes e perfurocortantes quanto para o comum;

- Aquisição de carros de transporte adequados para o transporte interno dos resíduos.

Sabe-se, contudo que o amadurecimento do processo virá com o tempo; todavia, é necessário integrar tais propostas na política interna da organização, de modo que possa envolver todos os níveis organizacionais, desde os colaboradores da base até a Mesa Administrativa da unidade hospitalar.

\section{REFERÊNCIAS}

ABNT - ASSOCIAÇÃO BRASILEIRA DE NORMAS TÉCNICAS. NBR - 12807: Resíduos de serviços de saúde - terminologia. Rio de Janeiro, 1993.

AMANTHEA, E; BELLI, R; DAMASCENO, J. W; JUNIOR, C. B; LAZARIN, F. B; TSUJIOKA, R. Y. D; XAVIER, G. A. Gerenciamento de Resíduos de Serviço de Saúde. In: VI Congresso Brasileiro de Engenharia Química em Iniciação Científica, 2005, Campinas. Anais... Campinas: Universidade Estadual de Campinas - UNICAMP, 2005.

ANVISA - AGÊNCIA NACIONAL DE VIGILÂNCIA SANITÁRIA. Resolução no 306 de 07 de dezembro de 2004. Regulamento técnico para o gerenciamento de resíduos de serviços de saúde. Diário Oficial da República Federativa do Brasil, Brasília, DF, 10 dez. 2004.

(BRASIL). MINISTÉRIO DA SAÚDE. AGÊNCIA NACIONAL DE VIGILÂNCIA SANITÁRIA. Manual de gerenciamento dos resíduos de serviços de saúde. Brasília, Ministério da Saúde, 2006.

(BRASIL). MINISTÉRIO DA SAÚDE. Saúde ambiental e gestão de resíduos de serviços de saúde. Brasília-DF: Ministério da Saúde, 2002. 
BRITO, M. A. G. de M. Considerações sobre resíduos sólidos de serviços de saúde. Rev. Eletr. de Enfermagem, 2 (2), UFG, 2000.

CAMARGO, M. E; MOTTA, M. E. V; LUNELLI, M. O; SEVERO, E. A. Resíduos sólidos de serviços de saúde: um estudo sobre o gerenciamento. Scientia Plena, v. 5. n. 7, 2009.

CATÃO, G. C; NETO, J. D; FARIAS, M. S. S. de; DANTAS, T. B. Diagnóstico e análise do gerenciamento dos resíduos hospitalares da cidade de Campina Grande - Paraíba. Hygeia 3 (5), dez. 2007.

COSTA, W. M. da; FONSECA, M. C. G. da. A importância do gerenciamento dos resíduos hospitalares e seus aspectos positivos para o meio ambiente. In: HYGEIA - Revista Brasileira de Geografia Médica e da Saúde, 5 (9), dez./2009.

FERREIRA, J. A. Resíduos sólidos e lixo hospitalar: uma discussão ética. Cad. de Saúde Pública, Rio de Janeiro, 11 (2), abr./jun, 1995.

GARCIA, L. P; ZANETTI-RAMOS, B. G. Gerenciamento dos resíduos de serviços de saúde: uma questão de biossegurança. Cad. de Saúde Pública, Rio de Janeiro, 20 (3), mai./jun. 2004.

IBAM/SEDU. Manual de gerenciamento integrado de resíduos sólidos. Rio de Janeiro: IBAM, 2001. Disponível em http://www.ibam.org.br/publique/media/manuallRS.pdf. Acesso em 25 mar. 2010.

LIPPEL, M. Modelo de gerenciamento de resíduos sólidos de saúde para pequenos geradores - o caso de Blumenau/SC. 2003. 120f. Dissertação (Mestrado). Programa de Pós-Graduação em Ciência e Engenharia de Produção. Universidade Federal de Santa Catarina - UFSC. Florianópolis, 2003.

MARANGONI, M. C. Gerenciamento de serviço de resíduo de saúde: estudo de caso no hemocentro da Unicamp. 2006. 114f. Dissertação (Mestrado). Faculdade de Engenharia Civil, Arquitetura e Urbanismo. Universidade Estadual de Campinas, UNICAMP. Campinas-SP, 2006.

NAIME, R; RAMALHO, A.H. P; NAIME, I.S. Avaliação do sistema de gestão dos resíduos sólidos do Hospital de Clínicas de Porto Alegre. Revista Espaço para a Saúde, v. 9, n. 1, Londrina-PR, dez/2008.

ORGANIZAÇÃO PAN-AMERICANA DE SAÚDE. Centro Pan-Americano de Engenharia Sanitária e Ciências do Ambiente. Guia para o manejo interno de resíduos sólidos em estabelecimentos de saúde. Brasília, DF: OPAS, 1997. 
PEREIRA, L. C; TOCCHETTO, M. R. L. Sistema de gestão e proteção ambiental. São Paulo, 2004.2 Disponível em: $<$ http://ambientes.ambientebrasil.com.br/gestao/artigos/sistema de gestao e protecao a mbiental.html>. Acesso em 20 abr. 2010.

SANCHES, P. S. Caracterização dos riscos nos resíduos de sistema de saúde e na comunidade. In: Gerenciamento de resíduos sólidos de serviços de saúde. São Paulo: CETESB, 1995.

SILVA, L. T. de C. V. da. Caracterização do resíduo hospitalar: uma inferência à patogenicidade. 1993. 154 f. Dissertação (Mestrado em Engenharia Civil) - Faculdade de Engenharia Civil. Universidade Estadual de Campinas. Campinas/SP, 1993.

TAKAYANAGUI, A. M. M. Gerenciamento de resíduos de serviços de saúde. In: PHILIPPI JR. A. (editor). Saneamento, saúde e ambiente: fundamentos para um desenvolvimento sustentável. Barueri-SP: Manole, 2005.

TEIXEIRA, G. P. A gestão dos resíduos de serviços de saúde no município de Juíz de Fora-MG. In: VIII Seminário Nacional de Resíduos Sólidos. ABES/MA - Seção Maranhão da ABES, 2006. 